\title{
Assessing Fidelity to and Satisfaction with the "Adolescent Coping with Depression Course" (ACDC) Intervention in a Randomized Controlled Trial
}

\section{Serap Keles ${ }^{1}\left(\mathbb{0} \cdot\right.$ Görel Bringedal $^{2} \cdot$ Thormod Idsoe $^{2}$}

Accepted: 10 October 2021 / Published online: 2 November 2021

(c) The Author(s) 2021

\begin{abstract}
This paper aims at describing the process for assessing the intervention fidelity of a randomized controlled trial (RCT) of an "Adolescent Coping with Depression Course" (ACDC) and to assess the participants' satisfaction with the intervention. We applied the comprehensive fidelity model developed by the National Institutes of Health's Behavior Change Consortium to examine how our intervention met the fidelity requirements under five categories. Data came from a two-arm parallel cluster RCT. Both qualitative and quantitative analyses of the ACDC intervention using the comprehensive fidelity model indicated that the level of fidelity in this study did not reach $100 \%$. However, it was approaching a high level of treatment fidelity. Participants also expressed high levels of satisfaction $(M=3.65, S D=.95)$. This analysis is important to show how appropriately the intervention was implemented, areas for improvement to increase its fidelity, and to ensure the internal and external validity of the findings. Trial Registration: ISRCTN registry ISRCTN19700389. Registered 6 October 2015. https://doi.org/10.1186/ISRCTN19700389. Full Protocol: https://doi.org/10.1186/s12888-016-0954-y
\end{abstract}

Keywords Intervention fidelity - Satisfaction with intervention · Adolescent depression · Group CBT · Cluster RCT

Serap Keles

serap.keles@uis.no

Görel Bringedal

g.e.bringedal@nubu.no

Thormod Idsoe

thormod.idsoe@nubu.no

1 Knowledge Center for Education, University of Stavanger, Forus, P.O. Box 8600, 4036 Stavanger, Norway

2 Norwegian Center for Child Behavioral Development, Majorstuen, P.O. Box 7053, 0306 Oslo, Norway 
Intervention fidelity refers to the extent to which interventions are delivered as intended in line with the intervention protocol or manual (Borrelli, 2011; Mowbray et al., 2003). Assessing intervention fidelity is essential when drawing conclusions on intervention effects, otherwise we cannot know whether potential findings may be attributed to the intervention itself or to other factors manipulated during the implementation process (Borrelli, 2011). Our purpose was to evaluate the fidelity of a randomized controlled trial (RCT) of a Cognitive Behavioral Therapy (CBT) based "Adolescent Coping with Depression Course" (ACDC) (Børve, 2010; Idsoe \& Keles, 2016) and to assess the participants' satisfaction with the intervention.

To interpret correctly an intervention effect or lack of it, we need to ensure that the intervention was carried out as designed (Perepletchikova \& Kazdin, 2005). In addition, for study replication and for generalization of interventions to real-world settings, the intervention fidelity has to be established (Borrelli et al., 2005). Hence, improving intervention fidelity has the effect of increasing both internal and external validity (Borrelli et al., 2005). Moreover, research shows that fidelity is significantly associated with the outcomes achieved by a program/ intervention (Durlak \& DuPre, 2008). In their review, which was based on several meta-analyses that, together, had reviewed more than 500 implementation studies targeting children and adolescents, Durlak and DuPre (2008) found that wellimplemented programs with high fidelity achieved effect sizes three times greater than poorly implemented programs.

Adherence to core program as specified in intervention manuals and competent delivery of the intervention have often been argued to be the two important dimensions of fidelity assessment (Forgatch \& DeGarmo, 2011). However, a more comprehensive treatment fidelity framework with five components was identified by the National Institutes of Health's (NIH) Behavior Change Consortium: (a) study design (i.e., factors considered when designing, evaluating and replicating a trial), (b) provider or facilitator training (i.e., information about how the facilitators are trained and whether the training is standardized across facilitators), (c) treatment or intervention delivery (i.e., processes to improve and monitor delivery of the intervention to establish the delivery of the intervention as intended), (d) treatment or intervention receipt (i.e., processes ensuring whether the intervention participants understand the information provided), and (e) enactment of treatment or intervention (i.e., processes to monitor and improve how participants use the skills from the interventions in their lives) (Bellg et al., 2004; Borrelli, 2011; Borrelli et al., 2005). Furthermore, NIH Behavior Change Consortium recommends that "treatment fidelity should become an integral part of the conduct and evaluation of all health behavior intervention research" (Bellg et al., 2004, p. 451). Hence, in this paper, the purpose was to examine the intervention fidelity of the intervention across each of these five domains. In addition, we assessed the participants' satisfaction with the intervention, since satisfaction may be considered one of the crucial validators of the quality of an intervention.

The "Adolescent Coping with Depression Course" (ACDC), which was developed for the Norwegian context, is a CBT-based group course for adolescents with subclinical or mild to moderate depression (Børve, 2010). The ACDC intervention has recently been evaluated using an RCT design (Idsoe \& Keles, 2016; Idsoe et al., 
2019). The results showed a small to medium reduction in depressive symptoms for the ACDC group compared to the usual care (UC) control group $(\mathrm{d}=-0.31)$.

This study is a part of the evaluation of the ACDC intervention (Idsoe \& Keles, 2016; Idsoe et al., 2019). It is necessary to assess implementation fidelity to gain a comprehensive evaluation of an intervention. In this paper, we examined fidelity to and satisfaction with the intervention. More specifically, we evaluated (a) the degree of intervention fidelity in five domains, and (b) the degree of perceived satisfaction with the ACDC intervention, assessed using reports from the participants. Because of lack of fidelity assessment in the control group, this article limits its focus to the fidelity of the ACDC intervention and to the satisfaction of the intervention participants.

\section{Method}

Data came from a two-arm (ACDC-Adolescent Coping with Depression Course vs. UC-Usual Care) parallel cluster randomized controlled trial (RCT), with course leaders as the unit of allocation and youth participants as the unit of analysis. Further details regarding study design, recruitment, inclusion/exclusion criteria, intervention content, attrition, and missing data mechanisms have been extensively described in elsewhere (Idsoe \& Keles, 2016; Idsoe et al., 2019), and will therefore only briefly be summarized throughout the text (see also Appendix for more details). Figure 1 shows the participant flowchart in the original trial.

\section{Measures}

We applied the comprehensive fidelity model developed by the National Institutes of Health's (NIH) Behavior Change Consortium to examine how the CBT intervention as a case met the fidelity requirements under five categories: study design; training; intervention delivery; receipt of the intervention; and enactment of intervention skills. As a part of the detailed fidelity assessment and satisfaction outcome, quantitative data consisted of intervention facilitators' ( $N=18,100 \%$ female) self-report assessments of intervention delivery, and the participants' $(N=133,91 \%$ girls; Mean $_{\text {Age }}=16.55$ years, $S D=1.10$ ) satisfaction with the intervention.

\section{Fidelity-Intervention Delivery}

Two measures of intervention fidelity were collected from the course facilitators: coverage of core components and form of delivery. The first scale consisted of six items on the core components of ACDC, themes covered in the ACDC manual. The ACDC facilitators rated the extent to which they covered each topic, such as emotion regulation, during the delivery of the intervention. The second scale consisted of five items assessing the degree to which the facilitators used different forms of delivery of the intervention, such as using video clips and examples from the web. In addition to the questions measuring these two aspects of intervention delivery, 


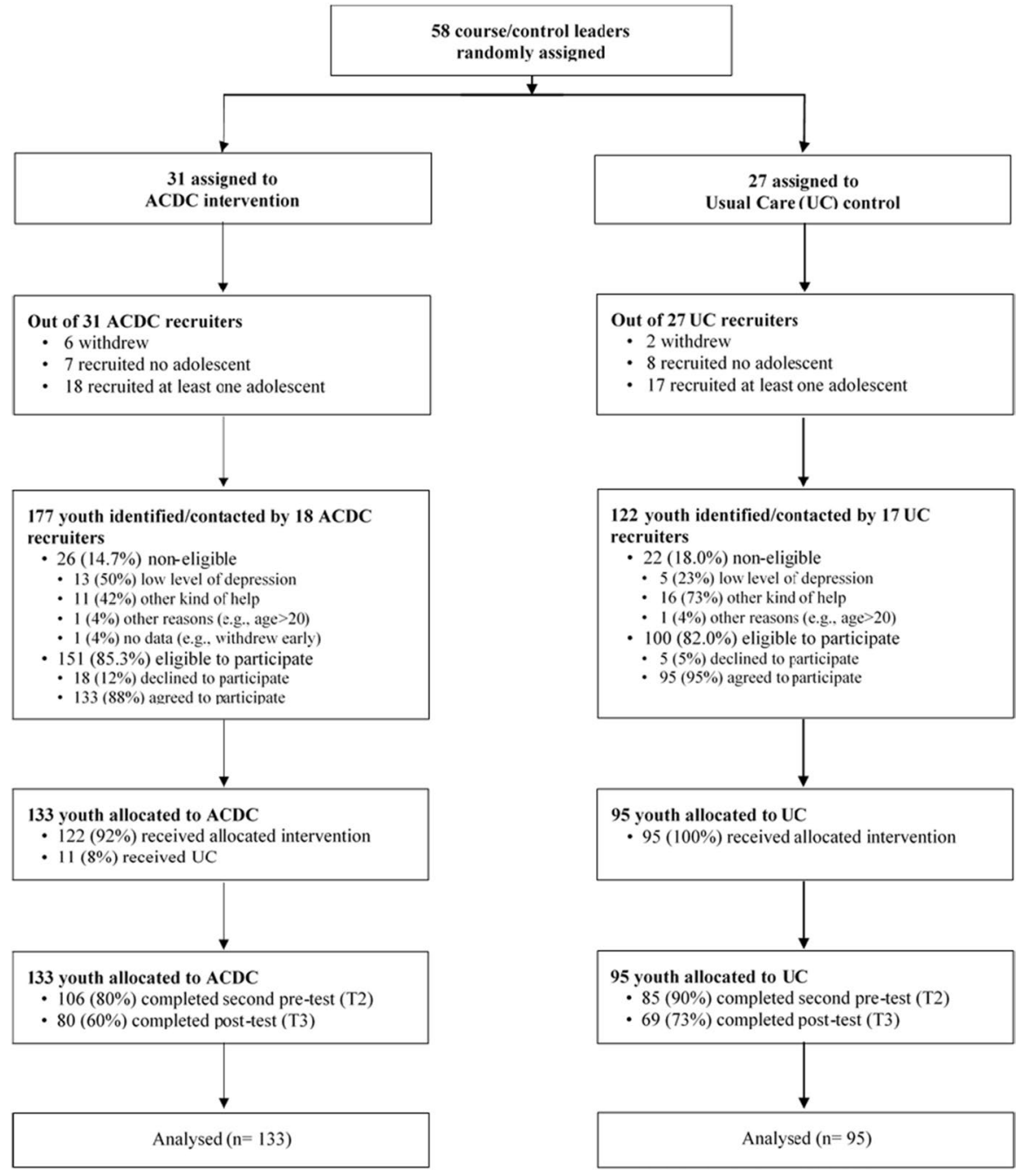

Fig. 1 Participants flowchart

one general question was asked: "To what extent do you think you followed the ACDC manual?" Each of the above items were rated on a scale from 1 (never) to 4 (most of the time). The content of these items was developed in collaboration with the course developer.

\section{Intervention Satisfaction}

To evaluate satisfaction with the intervention, the participants completed a 8 -item brief satisfaction survey, which was the modified version of the scale developed by Ginsburg and Drake (2002), This original scale was a 7-item measure to evaluate 
treatment satisfaction of a school-based group CBT for anxiety disorders. This measure covered several aspects considered as important to investigate for the ACDC program, such as perceived satisfaction with the program overall, the information given during sessions, out of session assignments, helpfulness of the therapists, being in a group, and so on. These items were modified to fit with our depression course and context, see Table 2 for an overview of the items. The ACDC intervention group of adolescents was asked these questions, rated on a scale from 1 (strongly disagree) to 5 (strongly agree).

\section{Procedure}

Data were collected via self-reported questionnaires. The measures provided in this manuscript were collected after the intervention period ended. The participants received electronic post-intervention questionnaires to report their level of satisfaction with the intervention along with the other study measures. Following the completion of the intervention, we also gathered information from the course facilitators on intervention delivery.

\section{Ethical Statement}

This investigation has been performed in accordance with the Declaration of Helsinki, and has been approved by The Norwegian Regional Committee for Medical and Health Research Ethics (South East). Approval reference: 2015/1027 Depresjonsmestring for ungdom. All the participants signed the consent form. They were also informed that participating in the study was voluntary and that participants could at any time and without any given reason withdraw their consent to participate. This would not affect their further participation on the course or in the usual care treatment. Contact information was provided for these issues.

\section{Results}

\section{Study Design}

The first core component for intervention fidelity is related to the study design, and consists of factors that should be considered when planning a trial as well as those that should be reported in order to evaluate and allow replication of the trial (Bellg et al., 2004; Borrelli, 2011). Initially, the design of the intervention should be guided by theory (Rew et al., 2018). ACDC is based on a new and updated understanding of depression within CBT, and the program contains different approaches and methods taken primarily from the work of Rational Emotive Behavior Therapy (REBT) (Ellis \& Grieger, 1977) and Cognitive Behavioral Therapy (CBT) (Beck et al., 1979). ACDC also includes elements from (a) Meta-Cognitive Theory (MCT) (e.g., learning how to modify the experience and regulation of your own thoughts, how to reflect on your own thinking style within a meta-perspective) (Wells et al., 2009), 
(b) Positive Psychology (PP) (e.g., emphasizing awareness and training in breaking thought patterns) (Seligman, 2006) as well as (c) modern neurobiological perspectives (e.g., understanding of how information is processed and why we react the way we do, sometimes automatically) (Børve, 2010). With this conceptual understanding, the adolescents can root their work with the concrete techniques in a solid base.

The standardized ACDC manual, which is printed and published by the Norwegian Council of Mental Health, has a detailed description of the active components of the intervention as well as the dose and duration. The course is delivered in a group format over eight consecutive weekly sessions, each lasting approximately $120 \mathrm{~min}$, including breaks. Two follow-up sessions are conducted approximately three and six weeks after the eighth session, lasting approximately $90 \mathrm{~min}$. In total, the ACDC consists of 10 sessions (see Garvik et al., 2014 for detailed descriptions of each session). During the evaluation study, the course facilitators received online questionnaires after each weekly session to report the dosage and involvement of the participants. On average, the adolescents receiving the ACDC intervention attended 6.5 of the ten sessions.

To monitor and enhance theoretical fidelity, Borrelli (2011) further suggests the strategy of reviewing the protocol and intervention manuals by an expert committee to ensure that the study design is operationalized as hypothesized. The design of ACDC intervention has also been reviewed by "Youngmind" (Ungsinn: http://www. ungsinn.no), which is a database for evidence-based interventions in treatment, prevention and health promotion in child and adolescent mental health, in Norway. The review group (Wergeland et al., 2016) concluded that the ACDC intervention is based on a well-founded theory, is well described in several documents including a detailed manual, and its aims and target groups are well defined.

\section{Facilitator Training: Eligibility and Training of Course Facilitators}

The training of the intervention facilitators should be standardized to ensure intervention fidelity (Beck et al., 2015). The level of credentials and years of experience required for intervention providers should also be decided a priori (Bellg et al., 2004). As mentioned, to become a certified ACDC course facilitator, individuals must have a minimum of 3-year college/university education (e.g. psychology, education, health, or related disciplines) before attending the 5-day certification course. The course facilitators, who can be medical doctors, psychologists, nurses, teachers, social workers, and so on, are employed in a workplace with a referral system such as community health, public health/public hospitals, schools or in private healthcare. The eligible individuals must attend a five-day intensive training program to be a certified course facilitator. The course developer, who is a psychologist experienced in running ACDC certification courses and specialized in cognitive therapy, runs the training program that in total consists of $36 \mathrm{~h}$.

The ACDC facilitators then recruit adolescents with subclinical depression or mild-to-moderate depression from their local communities in Norway using different strategies like announcements in local newspapers, advertisements in social media and posts on selected and targeted webpages, traditional posters with information 
about the ACDC course, or by getting access via mental health professionals, school counselors, the Educational and Psychological Counseling Services, and the Children and Young People's Psychiatric Out-Patient Services.

In the ACDC evaluation RCT, 58 course facilitators ( $97 \%$ female) were recruited to participate in the study mainly via emailing directly all upper secondary schools in Norway, emailing all school health services, and educational follow-up services. Announcements about the evaluation study were also posted on strategic websites such as the webpage of Norwegian Council of Mental Health and in other different targeted social media. The course facilitators, who were mostly counselors/special educators at school (44\%), school/health nurses (26\%), social workers (15\%), and psychologists $(12 \%)$, were randomized to experimental $(\mathrm{k}=31)$ and control $(\mathrm{k}=27)$ conditions by research administrative personnel at the Norwegian Center for Child Behavioral Development. ACDC facilitators received their training for a full week $(36 \mathrm{~h})$ before the recruitment of the adolescents and the intervention period started, while the UC facilitators received a one-day training in how to recruit eligible adolescents in order to standardize the recruitment process. Of the 31 ACDC facilitators, 30 completed the certification.

Out of 58 course facilitators, eight withdrew at the beginning of the study period, while 15 recruited no adolescents and were thereby not able to run ACDC or UC. Finally, 18 facilitators randomized to the experimental condition and 17 facilitators randomized to the control condition were included in the study (see Fig. 1).

\section{Intervention Delivery}

The "intervention delivery" category emphasizes processes to maintain and improve the intervention delivery as intended (Borrelli, 2011). One of the most crucial strategies that needs to be included to ensure the intervention delivery in terms of its content and dose as originally conceptualized, is to use an intervention manual (Borrelli, 2011; Rew et al., 2018). As identified before, the ACDC facilitators were trained to follow the protocol manual to ensure that all the participating adolescents received the same information and practiced the same skills during the intervention. Ongoing coaching and feedback for facilitators is an important way of monitoring intervention delivery. Therefore, during the intervention period, the course facilitators could keep in contact with the course developer through a closed social media group set up to address issues and get further feedback related to the delivery of the intervention as intended, and also by directly contacting the course developer.

In fidelity assessment, coverage of specific components of the intervention, accuracy and consistency of the implementation of each component, and the competence of the facilitator, are often assessed using self-reported measures completed by the facilitator themselves, or by ratings given by trained observers. Even though direct observation of facilitators' behavior is considered the gold standard for measuring fidelity to psychosocial interventions, this method requires extensive resources (Beidas et al., 2016) which were not available to us. At the end of the intervention period, the course facilitators provided information on how well they thought they 


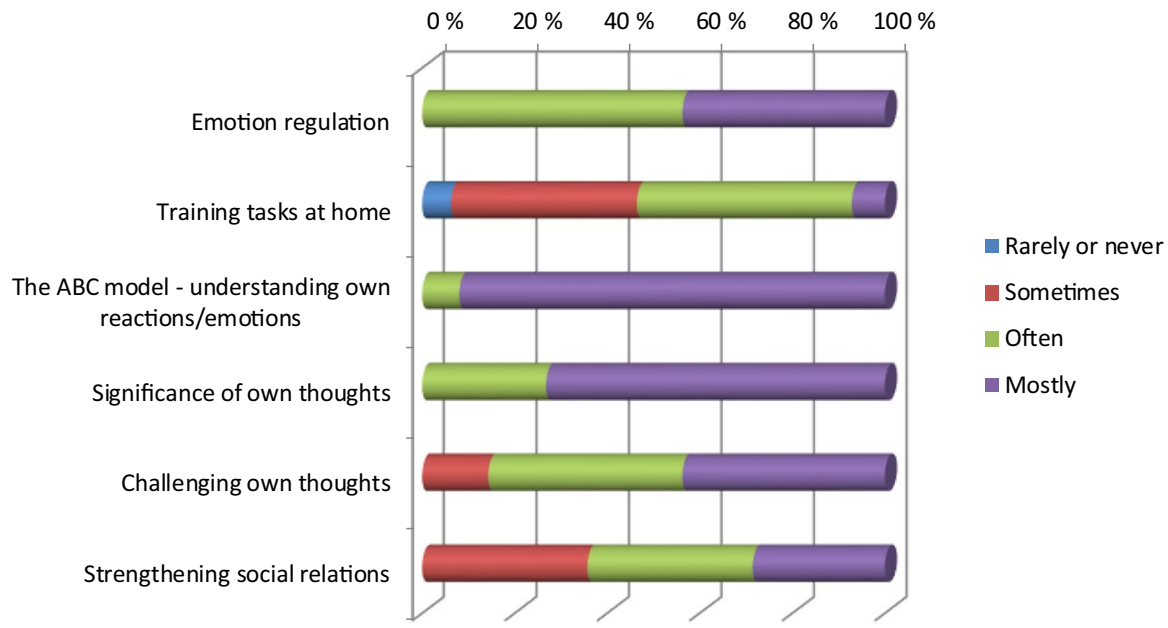

Fig. 2 The coverage of the core components of ACDC (ACDC Facilitators N=18)

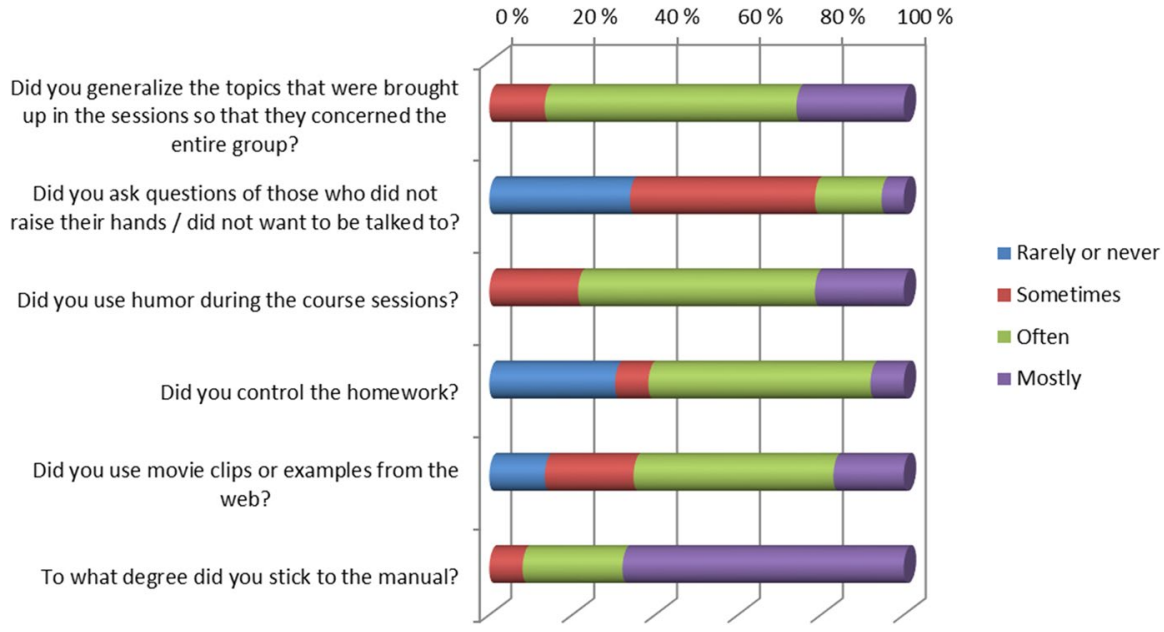

Fig. 3 The form of delivery (ACDC Facilitators $\mathrm{N}=18$ )

had delivered the intervention throughout the study (i.e., coverage of core components, and form of delivery), through self-report questionnaires.

In terms of specific manual content, Fig. 2 gives an overview of the ACDC facilitators' ratings on their adherence to the manual for each item separately. The frequency distributions for the content of delivery items were mostly rightly skewed; that is ACDC facilitators were more likely to report either "often" or "most of the time". For only one item (i.e., training tasks at home), the category "never" was reported by $6 \%$. In terms of form of delivery of the intervention, the frequency distribution, compared to the adherence scale, was less rightly skewed (see Fig. 3). The most frequently used form of delivery was the use of humor during the course 
sessions, while the category "never" was reported by $30 \%$ of the facilitators for the item with regard to reviewing the adolescents' homework, which is supposed to be reviewed at the beginning of each session. Interestingly, one item was developed by the course developer as a 'not-to-do' thing during the delivery of the sessions. "Did you ask questions of those who did not raise their hands/did not want to be talked to?" During the training, the facilitators were instructed not to address directly those who did not raise their hands. However, fewer than $34 \%$ of the course facilitators reported they "never" did this, while $45 \%$ of them reported they did this "sometimes" and more than $20 \%$ "often" and "most of the time".

\section{Intervention Receipt}

The domain of intervention receipt mainly focuses on whether the treatment delivered was actually received by the participants (Borrelli et al., 2005). This covers assessment of the participant's understanding of the intervention as well as their ability to use the skills acquired during the intervention. While intervention delivery is about 'what is actually taught', intervention receipt is about 'what is learned' (Borrelli et al., 2005).

In the ACDC manual, the importance of practicing the techniques learned from the course is emphasized both in and outside the course setting to learn and develop the necessary skills throughout the intervention. One of the goals of the course is for the adolescents to acquire a 'toolbox' of skills and techniques to help them cope better with their depressive symptoms in the future. Especially in the last three weekly sessions, the focus is on encouraging participants to practice new skills taught in the sessions. Each subject area, such as coping with distorted cognitions, is practiced by meeting various situations with applicable techniques (Garvik et al., 2014).

Lastly, in the weekly dosage reports, the course facilitators also provided ratings for each participant for their involvement in each session. The engagement of the participants during the sessions may indirectly reflect the degree of their intervention receipt in terms of understanding and learning the concepts and skills covered in the sessions. On average, course facilitators reported that $75 \%$ of the adolescents were involved in the sessions.

\section{Enactment of Intervention}

Enactment of the intervention skills acquired during the intervention (i.e., performing intervention-related behavioral skills and cognitive strategies in relevant real-life settings) is the final and most important component of the evaluation of intervention fidelity (Bellg et al., 2004; Borrelli, 2011; Borrelli et al., 2005). Even though the enactment component is sometimes equated with the findings of a study such as the effectiveness of the intervention (Rew et al., 2018), NIH Behavior Change Consortium conceptualized it as an aspect of implementation. Compared to 'what is taught' (delivery) and 'what is learned' (receipt), enactment is 'what is actually used' by the participants (Borrelli, 2011). 
In addition to the emphasis on the importance of practicing the techniques learned on the course both in and outside the course setting, during the last two sessions of the ACDC intervention, the entire course is reviewed by asking the participants to share their daily experiences with the cognitive and behavioral techniques that they have been learning during the course. Adolescents were further asked to share and show enactment of specific skills covered in the intervention through role playing.

\section{Overall Fidelity Checklist}

To complete the fidelity evaluation, in addition to the examination above, we posthoc filled out the fidelity checklist of the use of treatment fidelity strategies developed by Borrelli et al. (2005) (see Table 1). Out of 25 items, 4 (16\%) of them were not applicable to this study because of the design with UC control condition. Of the remaining 21 fidelity checklist items, 15 (71\%) were identified in this study. According to Borrelli et al. (2005), this result indicates that the fidelity in this study was approaching a high level of treatment fidelity, which was suggested as $80 \%$.

\section{Satisfaction with the Intervention}

Table 2 displays the mean ratings for each of the items assessing the satisfaction with the intervention. Overall, participants expressed high levels of satisfaction $\left(M_{\text {satisfaction }}=3.65, \mathrm{SD}_{\text {satisfaction }}=0.95\right)$. The mean level of satisfaction across different facilitators ranged between 2.38 and 4.56 . The frequency distributions are rightly skewed, that is, most of the participants reported "agree" or "strongly agree". The highest satisfaction with the intervention was reported for the items "The course leader was helpful" $(M=4.41, S D=0.90 ; 83.6 \%$ agreed or strongly agreed), "I would recommend this course to others" $(M=4.16, S D=1.02 ; 77 \%$ agreed or strongly agreed) and "I am pleased with the location where the course was held" $(M=4.18, S D=1.01 ; 72.1 \%$ agreed or strongly agreed). The lowest satisfaction with the intervention, on the other hand, was reported for the items "I am pleased with the results of the course" $(M=3.57, S D=0.99 ; 57.4 \%$ agreed or strongly agreed) and "The training tasks in between sessions were helpful" $(M=3.59, S D=0.88$; $49.1 \%$ agreed or strongly agreed). Figure 4 also gives an overview of the ratings on each item of the satisfaction scale.

\section{Discussion}

This paper was a part of the evaluation of an RCT of the ACDC intervention that aims to decrease depressive symptoms among upper secondary school students (Idsoe \& Keles, 2016; Idsoe et al., 2019; Keles \& Idsoe, 2021). We applied the comprehensive fidelity model developed by the NIH's Behavior Change Consortium to examine how the implementation of the ACDC intervention met the fidelity strategies under five categories: study design; training; intervention delivery; receipt of the intervention; and enactment of intervention skills. We also evaluated 


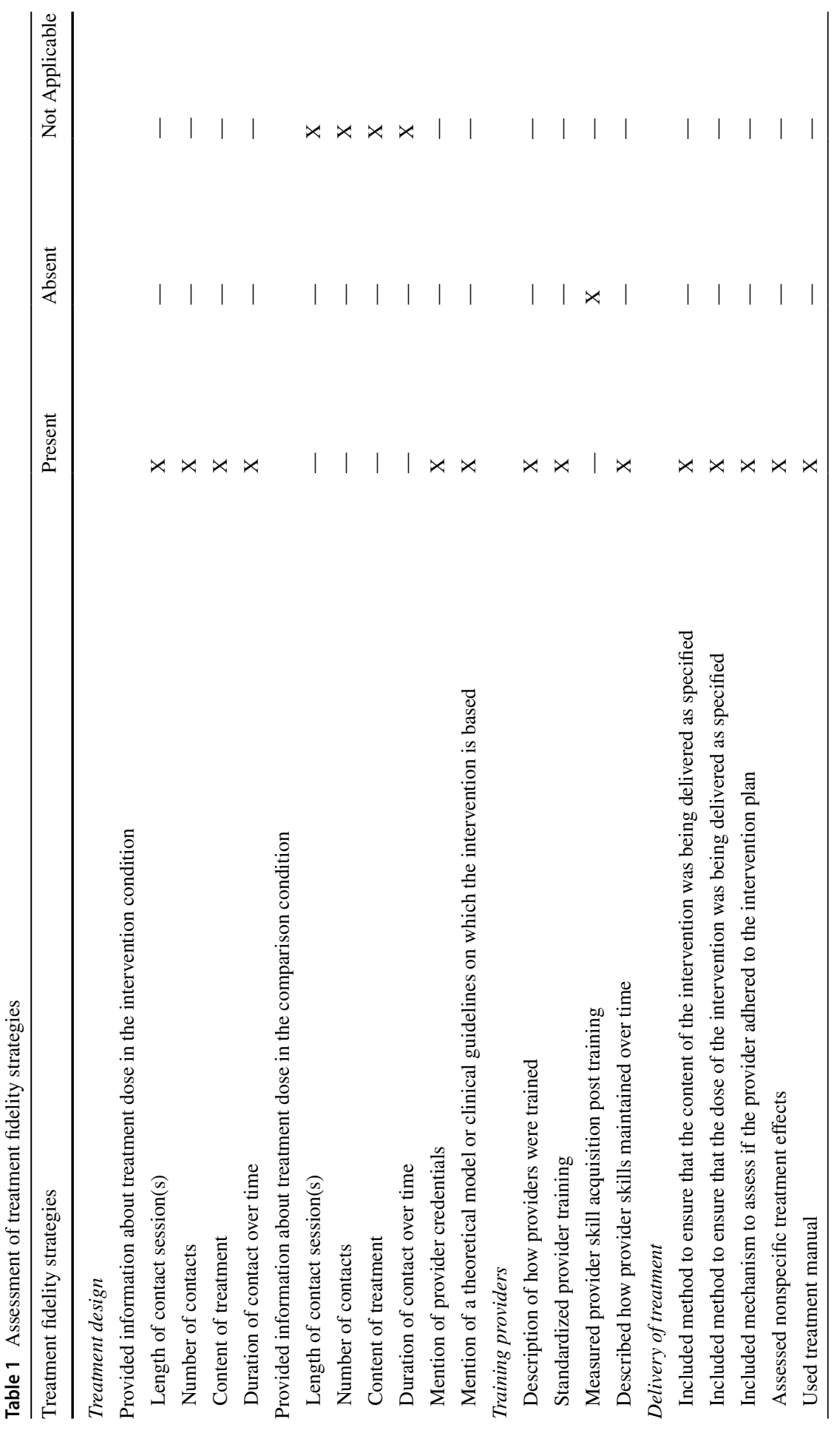




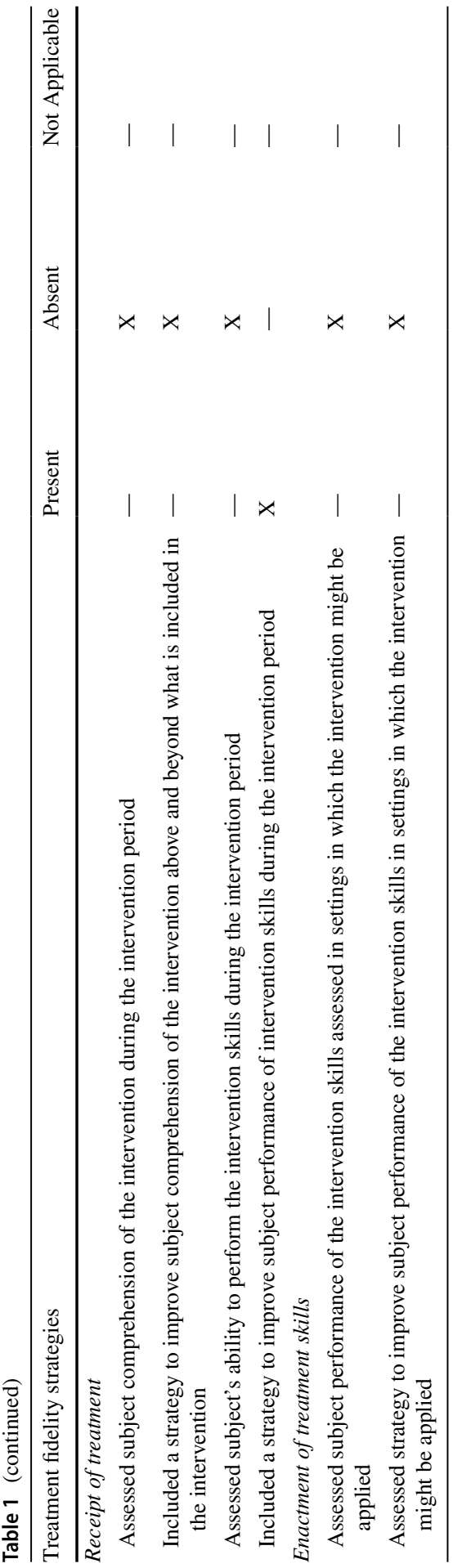


Table 2 Descriptives of the satisfaction with the intervention measure

\begin{tabular}{llc}
\hline & $\mathrm{M}$ & SD \\
\hline 1. This course was very helpful to me & 3.74 & 1.02 \\
2. I am pleased with the results of the course & 3.57 & .99 \\
3. I would recommend this course to others & 4.16 & 1.02 \\
4. The information we received during the course sessions was helpful & 3.98 & .96 \\
5. The training tasks in between sessions were helpful & 3.59 & .88 \\
6. The course leader was helpful & 4.41 & .90 \\
7. Being in a group and talking to others was helpful & 3.92 & 1.02 \\
8. I am pleased with the location where the course was held & 4.18 & 1.01 \\
\hline
\end{tabular}

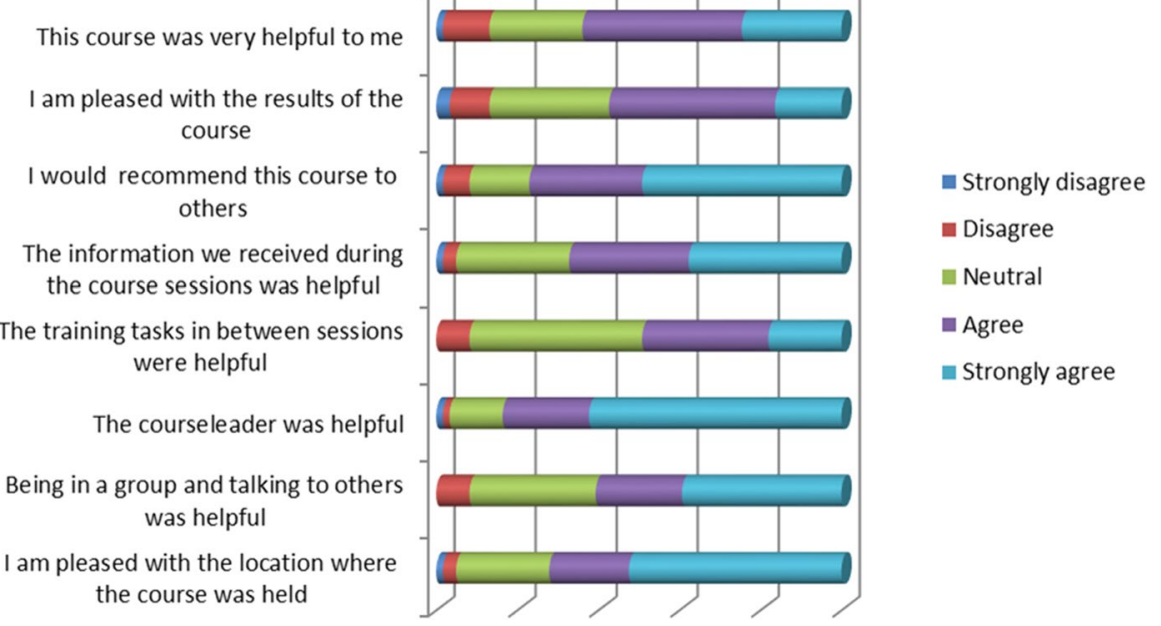

Fig. 4 Intervention satisfaction (ACDC Youth Participants N=133)

the participants' satisfaction with the intervention as one of the crucial validators of intervention quality.

Overall, our results revealed that the intervention achieved a fidelity of $71 \%$ according to the checklist of the use of treatment fidelity strategies developed by Borrelli et al. (2005). Our fidelity level was approaching that defined by Borrelli et al. (2005) as high fidelity, which was $80 \%$ adherence to their checklist across all strategies. A recent meta-analysis (Reiser \& Milne, 2014) also revealed that the included interventions achieved a mean overall level of $67 \%$ to the treatment fidelity framework, lower than the evaluation of the ACDC intervention. When we examined each of the five categories of the framework, in this trial we found out that the strategies under the design and the intervention delivery were more likely to be met. While some of the reviews also revealed that the most commonly reported elements 
in the fidelity literature are related to intervention design (Reiser \& Milne, 2014), other reviews showed that intervention delivery is the most commonly reported category while other categories are less discussed (Gearing et al., 2011; Slaughter et al., 2015).

With regard to the study design category, ACDC is based on a priori defined theoretical model, and a standardized manual to implement the intervention as intended; however, we still cannot ensure $100 \%$ that each participating adolescent received the same "operationalization" of the intervention on multiple sites. Hence, further strategies should be developed to enhance treatment fidelity related to the intervention study design.

In terms of facilitator training, the same trainer (i.e., the course developer) runs the training program and certifies the intervention facilitators to maintain standardization across intervention providers. However, as the intervention evaluators, we have limited information and control in terms of how skill acquisition both during and after training for the facilitators was assessed by the course developer in order to satisfy the criteria for certification. Moreover, even though there is a substantial focus on facilitator training at the beginning of intervention studies, there is less emphasis on monitoring and maintaining facilitator skills as studies progress (Bellg et al., 2004). In addition, most of the facilitators in this evaluation study were newly recruited so may not be highly skilled/experienced facilitators and this may limit the external validity of our findings. In future studies, facilitators' skill acquisition should be a part of the fidelity assessment of ACDC and strategies should be developed to minimize change or decay in facilitator skills. Examples of important skills could be pedagogical abilities to explain intervention content, securing intervention delivery as originally conceptualized, following the protocol, ensuring that all participants received the same information. This is important for treatment efficacy. A recent meta-analysis found that CBT for subclinical depression containing components like behavioral activation, challenging thoughts, and caregiver intervention gave better long-term outcomes for the adolescents (Oud et al, 2019). If there are differences in how facilitators prioritize among components, this could thereby correlate with intervention outcomes and affect clinical efficacy. In relation to this, in the ACDC manual, the use of co-facilitators in the delivery of intervention is suggested, even though it was not feasible owing to the limited resources in this study. This should be considered in future studies for enhancing fidelity.

For the delivery of the intervention, both the content and form of delivery were assessed by self-reports in this fidelity analysis. The measures of intervention delivery evaluated whether the facilitators actually adhered to the intervention plan in terms of both the content and the delivery form. The results revealed that ACDC facilitators were more likely to report either "often" or "most of the time" with regard to the coverage of core components of the intervention, however, some components were more emphasized than the others. Even though observation of intervention delivery is accepted as the gold standard to ensure acceptable delivery (Bellg et al., 2004) and even though self-report data are less correlated with intervention outcomes than observational data (Durlak \& DuPre, 2008), because of lack of resources, direct observation was not feasible in this study. At the study design stage, we, as the evaluators, considered using an observer to attend some of the 
sessions and evaluate adherence to the manual. However, this idea was discarded given the costs and time required across multiple sites in Norway. In future studies, more robust fidelity methods, such as independently rated audio or videotaping measures, should be considered.

The last two categories of the fidelity assessment focused on the participant rather than the facilitator. ACDC sessions not only focus on encouraging participants to learn and practice new skills taught in the sessions, but also to show enactment of specific skills through role playing. In the effectiveness evaluation of the intervention, we found changes in cognitive styles such as perfectionism and rumination after the intervention (Idsoe et al., 2019) and these findings may also indirectly support the acquisition and enactment of the participants' skills. On the other hand, during intervention delivery, $6 \%$ of the intervention facilitators never provided homework. This not only reflects issues regarding adherence to the manual, but it also limits our information regarding intervention receipt, since homework completion is a strategy suggested for assessing intervention receipt. Moreover, in their meta-analysis, Stice et al. (2009) showed that depression prevention programs with homework assignments produced significantly larger effects mainly because they provide increased opportunity to acquire and apply intervention skills in a real world setting. It is a limitation that we did not collect such data on homework completion, because this could have informed us whether homework completion is associated with improvement.

The satisfaction with the intervention was quite high and the participants perceived the course as helpful in their life and they would recommend the ACDC course to others in need. However, we are also aware of the fact that even though an adolescent participant might be very satisfied with the help s/he received in the intervention, it does not necessarily mean that s/he has actually learned the tools or techniques taught in the intervention or applied them in his/her lives. A short followup of the participants with regard to the degree they use the techniques and tools they learned during the intervention in their lives after the intervention ended would contribute to validity of our results. Further studies should also use more qualitative data methodology (e.g., open-ended questions) to get in-depth information on intervention satisfaction. One issue here is that the participants' reports of their satisfaction could have been biased by the fact that they did not report this anonymously, that especially may have affected their willingness to report dissatisfaction. Future studies should try to reduce this potential methodological limitation.

This analysis has its own limitations. One of the most important limitations is the lack of fidelity assessment in the control group in our evaluation study. Borelli (2011) suggests that we cannot see the true differences between intervention and control groups without monitoring the fidelity in the UC control group. However, in our evaluation study, participants in the control group received usual care as implemented at the different sites. This may involve referrals to very different care providers such as psychologists, school nurses, or medical doctors who may provide conversations, various standard treatments, the use of pharmacotherapy or no treatment at all. Hence, in the evaluation study, the UC facilitators and the adolescents in the UC group were asked to report who they were referred to and who they received care from. However, it was not possible to develop a structured fidelity scheme 
for this heterogeneous group, since no restrictions were put on what kind of interventions the adolescents in the control group could receive. Another limitation in the evaluation study was in relation to the group size for each course group. In the manual of this group CBT-based course, it is specified that a group should consist of about 8-12 participants for each course since it is important that the group is not too small in terms of group dynamics (Børve, 2010). The average group size in the evaluation study was six, and this should be kept in mind while evaluating both the intervention delivery from the course facilitators' angle and intervention receipt from the participants' angle. Moreover, according to the dosage reports, on average, the adolescents receiving the ACDC intervention attended 6.5 of the ten sessions. With regard to fidelity, especially for the enactment of the skills aspect, we checked weekly dosage reports post-hoc to examine whether there is a pattern in terms of missing last sessions (where the focus is mainly on practicing the new skills) or in certain clusters. Fortunately, there was no clear pattern, but we still cannot rule out the possibility that for some participants this may have occurred (i.e., missing the last sessions). However, the lack of a pattern is good in terms of the effectiveness of the intervention, since that could have led to under-estimation of the intervention effect.

Research also shows that higher levels of intervention fidelity are strongly associated with better intervention outcomes mainly because of reduction of unintended and random variability and increasing study power to detect the real effect (Borrelli et al., 2005; Durlak \& DuPre, 2008). In future studies, investigating the effect of core components and the effect of how they are implemented on study outcomes may provide more reliable and accurate results with regard to the validity and effectiveness of ACDC. We were not able to investigate these because of lack of power/ sample size.

Finally, yet importantly, there is an extensive debate on whether interventions should be implemented with maximum fidelity or whether adaptation should be encouraged (Durlak \& DuPre, 2008). As seen also in our case, studies showed that the levels of fidelity do not reach $100 \%$ and programs are modified during the implementation by providers (ibid.). Hence, this case-specific information on implementation fidelity also enables us to examine 'what works' or not in the real world setting to better inform the intervention implementation in long run and make necessary changes to improve/modify its implementation and examine how these modifications affect program outcomes.

In addition to fidelity, another important aspect of interventions is program reach, which is related to "the percentage of the eligible population who took part in the intervention, and their characteristics" (Durlak \& DuPre, 2008, p. 329). In the evaluation study of ACDC, one of the biggest challenges was with regard to the recruitment of the participants, especially boys. This may indicate that it may be difficult for adolescents, especially for boys, to admit that they have problems that they may need to seek help for. Alternatively, we may further speculate that since the course facilitators were mostly female, maybe it was harder for boys to ask for help. These recruitment challenges raise important issues not only about reaching those who need help but do not seek help, but also about starting interventions after recruiting enough participants to establish the minimum required group size. All of these 
issues may have had an effect on the outcomes and may affect our interpretation of the results. More proactive strategies are needed to reach both genders and these strategies should be part of the training of facilitators. Finally, future studies are also necessary to examine whether fidelity reduces over time.

\section{Conclusions}

We examined how the implementation of the ACDC intervention met the fidelity strategies under five categories (study design, training, intervention delivery, receipt of the intervention, and enactment of intervention skills), by using the comprehensive fidelity model developed by the NIH's Behavior Change Consortium, and we looked at the satisfaction level of participants. In line with other studies in the literature, the levels of fidelity in our analysis did not reach $100 \%$ and providers modified the intervention during the implementations. The domains of fidelity assessment which focus on the participant rather than the facilitator (i.e., receipt and enactment) should be prioritized in future fidelity evaluations. Future studies with more participants, better recruitment procedures, using more robust fidelity methods to check whether facilitators stick to the manual and assessing fidelity also in control groups are needed.

\section{Appendix}

\section{Detailed Information of the Study Method}

Data came from a two-arm (ACDC-Adolescent Coping with Depression Course vs. UC-Usual Care) parallel cluster randomized controlled trial (RCT), with course leaders as the unit of allocation and youth participants as the unit of analysis. Cluster randomization was chosen for practical reasons because of the recruiting process, and it reduced potential contamination between the two conditions. The first step in the recruiting process was to include and train potential course facilitators. To be eligible for the 5-day certification course, facilitators had to hold a minimum of 3-year college/university education (e.g. psychology, education, health, or related disciplines). Fifty-eight course facilitators were randomized to experimental $(\mathrm{k}=31)$ and control $(\mathrm{k}=27)$ conditions by administrative personnel at our research department. The facilitators for the intervention group received their training before the recruitment of adolescents and the intervention start, while the facilitators of the control condition received a one-day training in how to recruit adolescents in order to standardize inclusion procedures.

Adolescents with depressive symptoms were recruited by the ACDC/UC facilitators through various channels including by placing information in schools and health centers, providing information through GPs, advertisements in local newspapers, 
on websites for young people, information at the local hospital's medical meetings. They were screened for subclinical/ mild to moderate depression, according to the criteria of the DSM by the use of the Beck Depression Inventory (BDI) (A. T. Beck, Ward, Mendelson, Mock, \& Erbaugh, 1961) and had a brief clinical interview. Participants were recruited either by making direct contact themselves with course facilitators, or they were referred by health visitors, GPs, psychiatric clinics, municipal services, school counselors, and the like. The target population was students from the 1st or 2nd grade of upper secondary school, who were 16 and 17 years old. There was a maximum cut-off age of 20 years and subjects had to have subclinical depression or mild to moderate depression, according to the criteria of the DSM. Exclusion criteria included presence of bipolar disorder, psychosis, substance-use, ADHD or ADD and brain damage as listed in the ACDC manual. Language abilities good enough to follow the course were required. More details about training and inclusion is described in the results section, since the details were elaborated in the analyses part of the corresponding fidelity category.

ACDC participants received eight weekly sessions and two follow-up sessions approximately three and six weeks after the last session. UC participants received usual care as implemented at the different sites. In total, 228 adolescents (88\% girls; Mean $_{\text {Age }}=16.70$ years, $S D=1.14$ ) were recruited for the original trial.

Acknowledgements Thanks to Terje Ogden for his valuable suggestions in the final version of the manuscript.

Funding Open access funding provided by University Of Stavanger. Funder type: Research council. Funder name: The Research Council of Norway through the program "Research and Innovation in the Educational Sector" (FINNUT) (Project Number 238081/H20). Additional funding from Gidske og Peter Jacob Sørensens fond, and the Norwegian Directorate of Health. The development of the study protocol was supported by the Norwegian Center for Child Behavioral Development.

\section{Declarations}

Competing interests None of the authors has any competing interests.

Ethics Approval This investigation has been performed in accordance with the Declaration of Helsinki and has been approved by The Norwegian Regional Committee for Medical and Health Research Ethics (South East). Approval reference: 2015/1027 Depresjonsmestring for ungdom. All the participants signed the consent form. They were also informed that participating in the study was voluntary and that participants could at any time and without any given reason withdraw their consent to participate. This would not affect their further participation on the course or in the usual care treatment. Contact information was provided for these issues.

Consent to Participate Since the participants were above 16 years old, we did not obtain the consent from the parents or legal guardians of the participants, but participants themselves. This is in accordance with the Norwegian regulations, and thereby approved by The Norwegian Regional Committee for Medical and Health Research Ethics.

Consent to Publish Non applicable. No details, images, or videos relating to individual participants will be published.

Data Availability The datasets used and/or analysed during this study will be available from the corresponding author on reasonable request. 
Open Access This article is licensed under a Creative Commons Attribution 4.0 International License, which permits use, sharing, adaptation, distribution and reproduction in any medium or format, as long as you give appropriate credit to the original author(s) and the source, provide a link to the Creative Commons licence, and indicate if changes were made. The images or other third party material in this article are included in the article's Creative Commons licence, unless indicated otherwise in a credit line to the material. If material is not included in the article's Creative Commons licence and your intended use is not permitted by statutory regulation or exceeds the permitted use, you will need to obtain permission directly from the copyright holder. To view a copy of this licence, visit http://creativecommons.org/licen ses/by/4.0/.

\section{References}

Beck, A. K., Baker, A., Britton, B., Wratten, C., Bauer, J., Wolfenden, L., \& Carter, G. (2015). Fidelity considerations in translational research: Eating As Treatment-a stepped wedge, randomised controlled trial of a dietitian delivered behaviour change counselling intervention for head and neck cancer patients undergoing radiotherapy. Trials, 16(1), 465.

Beck, A. T., Rush, A. J., Shaw, B. F. H., \& Emery, G. (1979). Cognitive therapy of depression. Guilford Press.

Beidas, R. S., Maclean, J. C., Fishman, J., Dorsey, S., Schoenwald, S. K., Mandell, D. S., \& Hogue, A. (2016). A randomized trial to identify accurate and cost-effective fidelity measurement methods for cognitive-behavioral therapy: Project FACTS study protocol. BMC Psychiatry, 16(1), 323.

Bellg, A. J., Borrelli, B., Resnick, B., Hecht, J., Minicucci, D. S., Ory, M., \& Czajkowski, S. (2004). Enhancing treatment fidelity in health behavior change studies: Best practices and recommendations from the NIH Behavior Change Consortium. Health Psychology, 23(5), 443.

Borrelli, B. (2011). The assessment, monitoring, and enhancement of treatment fidelity in public health clinical trials. Journal of Public Health Dentistry, 71, s1.

Borrelli, B., Sepinwall, D., Ernst, D., Bellg, A. J., Czajkowski, S., Breger, R., \& Ogedegbe, G. (2005). A new tool to assess treatment fidelity and evaluation of treatment fidelity across 10 years of health behavior research. Journal of Consulting and Clinical Psychology, 73(5), 852.

Børve, T. (2010). Manual for Depresjonsmestring for ungdom [Manual for Adolescent Coping with Depression Course]. . Oslo: Rådet for psykisk helse (The Norwegian Council for Mental Health - NCMH).

Durlak, J. A., \& DuPre, E. P. (2008). Implementation matters: A review of research on the influence of implementation on program outcomes and the factors affecting implementation. American Journal of Community Psychology, 41(3-4), 327-350.

Ellis, A., \& Grieger, R. (1977). Handbook of rational-emotive therapy. Springer.

Forgatch, M. S., \& DeGarmo, D. S. (2011). Sustaining fidelity following the nationwide PMTO ${ }^{\mathrm{TM}}$ implementation in Norway. Prevention Science, 12(3), 235-246.

Garvik, M., Idsoe, T., \& Bru, E. (2014). Effectiveness study of a CBT-based adolescent coping with depression course. Emotional and Behavioural Difficulties, 19(2), 195-209.

Gearing, R. E., El-Bassel, N., Ghesquiere, A., Baldwin, S., Gillies, J., \& Ngeow, E. (2011). Major ingredients of fidelity: A review and scientific guide to improving quality of intervention research implementation. Clinical Psychology Review, 31(1), 79-88.

Ginsburg, G. S., \& Drake, K. L. (2002). School-based treatment for anxious African-American adolescents: A controlled pilot study. Journal of the American Academy of Child \& Adolescent Psychiatry, 41(7), 768-775.

Idsoe, T., \& Keles, S. (2016). Study protocol for a randomized controlled trial of a group cognitivebehavioral course for depressed adolescents. BMC Psychiatry, 16(1), 1-10. https://doi.org/10.1186/ s12888-016-0954-y

Idsoe, T., Keles, S., Olseth, A. R., \& Ogden, T. (2019). Cognitive behavioral treatment for depressed adolescents: Results from a cluster randomized controlled trial of a group course. BMC Psychiatry, $19,155$.

Keles, S., \& Idsoe, T. (2021). Six-and twelve-month follow-up results of a cluster randomized controlled trial of a CBT-based group course. Prevention Science, 22(4), 409-418. 
Mowbray, C. T., Holter, M. C., Teague, G. B., \& Bybee, D. (2003). Fidelity criteria: Development, measurement, and validation. The American Journal of Evaluation, 24(3), 315-340.

Oud, M., De Winter, L., Vermeulen-Smit, E., Bodden, D., Nauta, M., Stone, L., \& Kendall, T. (2019). Effectiveness of CBT for children and adolescents with depression: A systematic review and metaregression analysis. European Psychiatry, 57, 33-45. https://doi.org/10.1016/j.eurpsy.2018.12.008

Perepletchikova, F., \& Kazdin, A. E. (2005). Treatment integrity and therapeutic change: Issues and research recommendations. Clinical Psychology: Science and Practice, 12(4), 365-383.

Reiser, R. P., \& Milne, D. L. (2014). A systematic review and reformulation of outcome evaluation in clinical supervision: Applying the fidelity framework. Training and Education in Professional Psychology, 8(3), 149.

Rew, L., Banner, M., Johnson, K., \& Slesnick, N. (2018). Intervention fidelity and facilitator training. Western Journal of Nursing Research, 40(12), 1843-1860.

Seligman, M. E. P. (2006). Learned optimism: How to change your mind and your life. Vintage Books.

Slaughter, S. E., Hill, J. N., \& Snelgrove-Clarke, E. (2015). What is the extent and quality of documentation and reporting of fidelity to implementation strategies: A scoping review. Implementation Science, $10(1), 129$.

Stice, E., Shaw, H., Bohon, C., Marti, C. N., \& Rohde, P. (2009). A meta-analytic review of depression prevention programs for children and adolescents: Factors that predict magnitude of intervention effects. Journal of Consulting and Clinical Psychology, 77(3), 486-503. https://doi.org/10.1037/ a0015168

Wells, A., Fisher, P., Myers, S., Wheatley, J., Patel, T., \& Brewin, C. R. (2009). Metacognitive therapy in recurrent and persistent depression: A multiple-baseline study of a new treatment. Cognitive Therapy and Research, 33(3), 291-300.

Wergeland, G. J., Skotheim, S., \& Kvello, Ø. (2016). Kunnskapsoppsummering og klassifisering av tiltaket DU - Mestringskurs for ungdom (2.utg.). Ungsinn, 2(1), 1-14.

Publisher's Note Springer Nature remains neutral with regard to jurisdictional claims in published maps and institutional affiliations. 